\title{
Incidental MRI Findings of Acute Gadolinium Hypersensitivity
}

\author{
C. Amene ${ }^{a} \quad$ L.A. Yeh-Nayre ${ }^{b} \quad$ C.E. Dory ${ }^{c} \quad$ J.R. Crawford ${ }^{b, d}$ \\ Departments of ${ }^{\mathrm{a}}$ Neurosurgery, ${ }^{\mathrm{b}}$ Pediatrics, ${ }^{\mathrm{c}}$ Radiology and ${ }^{\mathrm{d}}$ Neurosciences, \\ University of California, San Diego, and Rady Children's Hospital, San Diego, \\ Calif., USA
}

\section{Key Words}

$\mathrm{MRI} \cdot$ Gadolinium · Allergy

\begin{abstract}
A 13-year-old girl with a remote history of juvenile pilocytic astrocytoma developed acute onset flushing, tachycardia and shortness of breath immediately following administration of gadopentetate dimeglumine during routine brain MRI that subsided following intravenous diphenhydramine. A retrospective review of the MRI results revealed multiple areas of contrast enhancement of the face, consistent with observed urticaria. The patient received pretreatment medications prior to subsequent gadolinium injections without incident. Gadolinium allergy is extremely rare and has been reported in less than $0.1 \%$ of injections. However, in patients who undergo anesthesia for MRI studies, similar subtle extracranial MRI findings should alert the neuroradiologist to possible gadolinium allergy that may warrant premedication prior to future injections.
\end{abstract}

\section{Introduction}

Gadolinium contrast agents have been safely used in children and adults for over 20 years. The incidence of gadolinium allergy in children is between 0.04 and $0.1 \%$, based on published case series [1-3]. In those patients with central nervous system tumors, gadolinium contrast injection is absolutely necessary to monitor disease status. We report a case of a 13-year-old girl, with a history of low-grade glioma, who developed shortness of breath and urticaria following injection of intravenous gadolinium while in the MRI scanner. The incidental MRI findings reported may assist in recognizing gadolinium allergy in younger patients who are under anesthesia during their neuroimaging studies. 


\section{Case Report}

A 13-year-old girl, diagnosed with a posterior fossa juvenile pilocytic astrocytoma, status post gross total resection 1 year prior, underwent routine MRI neuroimaging with and without gadolinium. Following an injection of intravenous gadopentetate dimeglumine, she developed acute onset flushing, tachycardia and shortness of breath. The patient was promptly removed from the MRI scanner and given intravenous diphenhydramine, after which she quickly returned to baseline. Her physical examination was notable for diffuse urticaria that resolved following treatment. Neurological examination showed no deficits. She had no prior history of allergic reactions and no known allergy risk factors. Brain MRI initially reported stable post-operative changes and no evidence of residual neoplasm. However, on retrospective review in light of the history, several extracranial areas of abnormality were discovered, as shown in fig. 1 . The post-gadolinium MRI revealed multiple areas of contrast-enhancing soft tissue lesions of the bilateral face consistent with the observed urticaria. Additionally, there was an area of contrast enhancement of the right ear. The patient has had subsequent MRI evaluations with gadolinium following pretreatment with corticosteroids and antihistamines without incident and with complete resolution of the aforementioned findings.

\section{Conclusions}

Our reported MRI findings of post-gadolinium hypersensitivity are admittedly coincidental; however, recognition of gadolinium allergy can be critical. Severe anaphylactoid reactions and even death have been well described in the literature [114]. Dillman et al. [1] reported a gadolinium allergy incidence of $0.4 \%$ in their pediatric population and a $7 \%$ overall incidence of severe reactions. Patients can have allergic reactions to gadolinium compounds in spite of pretreatment with antihistamines and corticosteroids [14]. Those patients with a prior history of allergic reaction to iodinated compounds may be at risk for reactions to gadolinium [15]. In the case of our patient, she had had several previous injections without incident and had no prior history of allergic reactions. Our case illustrates extracranial manifestations of gadolinium allergy that may alert the neuroradiologist, particularly in cases where patients are under anesthesia and may have less subtle findings.

\section{Disclosure Statement}

The authors have nothing to disclose and report no conflicts of interest. 

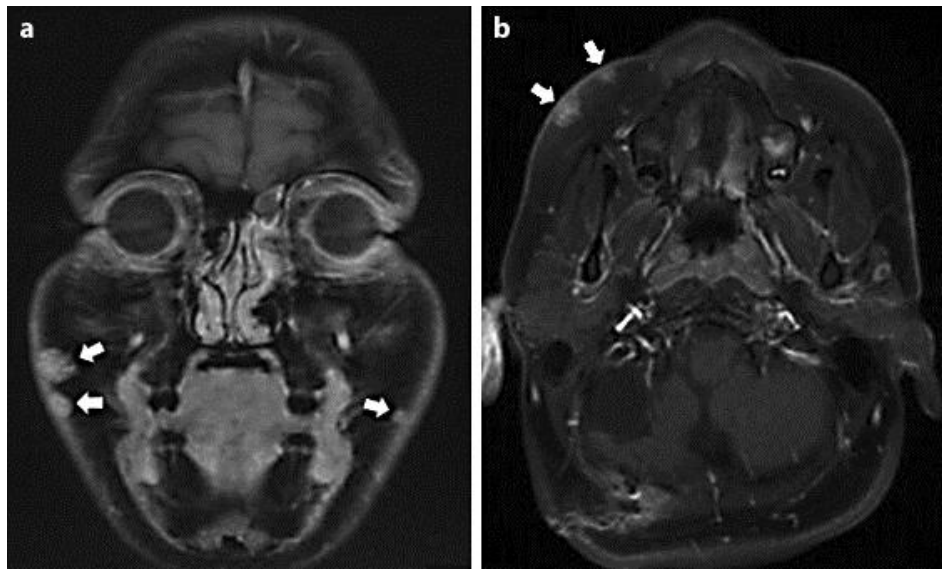

Fig. 1. MRI findings of gadolinium hypersensitivity. Coronal (a) and axial (b) post-contrast MRI brain sequences reveal multiple areas of superficial gadolinium enhancement (arrows) and right-sided auricular enhancement following administration of intravenous gadopentetate dimeglumine.

\section{References}

1 Dillman JR, Ellis JH, Cohan RH, Strouse PJ, Jan SC: Frequency and severity of acute allergic-like reactions to gadolinium-containing i.v. contrast media in children and adults. AJR Am J Roentgenol 2007;189:1533-1538.

-2 Murphy KJ, Brunberg JA, Cohan RH: Adverse reactions to gadolinium contrast media: a review of 36 cases. AJR Am J Roentgenol 1996;167:847-849.

-3 Hunt CH, Hartman RP, Hesley GK: Frequency and severity of adverse effects of iodinated and gadolinium contrast materials: retrospective review of 456,930 doses. AJR Am J Roentgenol 2009;193:1124-1127.

4 Bertherat J, Hoeffel C, Pariente-Khayat A, et al: Anaphylactic reactions after intravenous injection of gadolinium for pituitary or cerebral magnetic resonance imaging (in French). Presse Med 1996;25:2050.

-5 Jordan RM, Mintz RD: Fatal reaction to gadopentetate dimeglumine. AJR Am J Roentgenol 1995;164:743744.

-6 Kalogeromitros DC, Makris MP, Aggelides XS, et al: Anaphylaxis to gadobenate dimeglumine (Multihance): a case report. Int Arch Allergy Immunol 2007;144:150-154.

7 Lufkin RB: Severe anaphylactoid reaction to Gd-DTPA. Radiology 1990;176:879.

-8 Murphy KP, Szopinski KT, Cohan RH, Mermillod B, Ellis JH: Occurrence of adverse reactions to gadolinium-based contrast material and management of patients at increased risk: a survey of the American Society of Neuroradiology Fellowship Directors. Acad Radiol 1999;6:656-664.

-9 Salonen OL: Case of anaphylaxis and four cases of allergic reaction following Gd-DTPA administration. J Comput Assist Tomogr 1990;14:912-913.

10 Shepherd M, Lata S, Mani S, Fiorillo A, Kumar P: Anaphylaxis to gadolinium radiocontrast: a case report and review of the literature. J La State Med Soc 2009;161:282-284.

11 Simons CW, Benouni S, Gibbon G, Klaustermeyer W: Severe anaphylactoid shock secondary to gadolinium contrast media. Ann Allergy Asthma Immunol 2009;103:359-360.

-12 Singer BD, Woodrick RS, Pedicano JB: Severe adverse drug reaction to gadobenate dimeglumine. ScientificWorldJournal 2009;9:363-365.

13 Weiss KL: Severe anaphylactoid reaction after i.v. Gd-DTPA. Magn Reson Imaging 1990;8:817-818.

-14 Dillman JR, Ellis JH, Cohan RH, Strouse PJ, Jan SC: Allergic-like breakthrough reactions to gadolinium contrast agents after corticosteroid and antihistamine premedication. AJR Am J Roentgenol 2008;190:187-190.

15 Garcia N, Ramon E, Gonzalez del Valle L, Ruano M, Jimenez E: Importance of a previous allergy to an iodinated contrast agent in the administration of gadopentetate dimeglumine. Ann Pharmacother $1997 ; 31: 374$ 